Author-produced version of the paper published in EPL Europhisics letters, 2010 , vol.91, 58004.

Original publication available at

http://iopscience.iop.org/0295-5075/91/5/58004/

\title{
Opinion dynamics in a group-based society
}

\author{
F. Gargiulo ${ }^{1}$ and S. Huet ${ }^{1}$ \\ 1 LISC-Cemagref - 24 Avenue de Landais, Clermont Ferrand, France
}

PACS 89.65.-s - Social and economic systems

PACS 89.75. Fb - Structures and organization in complex systems

\begin{abstract}
Many models have been proposed to analyze the evolution of opinion structure due to the interaction of individuals in their social environment. Such models analyze the spreading of ideas both in completely interacting backgrounds and on social networks, where each person has a finite set of interlocutors. In this paper we analyze the reciprocal feedback between the opinions of the individuals and the structure of the interpersonal relationships at the level of community structures. For this purpose we define a group based random network and we study how this structure co-evolves with opinion dynamics processes. We observe that the adaptive network structure affects the opinion dynamics process helping the consensus formation. The results also show interesting behaviors in regards to the size distribution of the groups and their correlation with opinion structure.
\end{abstract}

Introduction. - Social science analyzes the large scale behaviour of the society. On the other side the interactions among the individuals are studied by social psychology. The main interest of social modeling is to create a bridge between the two levels of description, observing how, starting from the microscopic description of the interactions among the agents, the global phenomena observed in the society can be reproduced.

This kind of modeling has recently attracted the attention of the physicists both for the interest in the characterization of complex systems and for the rich critical behavior that such models exhibit.

In this paper we are going to focus on two main aspects observable in the society: the process of membership of groups and the process of opinion formation. The main rules of the interactions among the agents are dictated from social psychology. Other's opinion is a source of cognitive inconsistency! According to Festinger [8] such inconsistency is experienced as dissonance, a psychological discomfort that people are motivated to reduce.

Recently, [24] observed that this phenomenon is amplified inside the social groups. The groups are, in fact, suitable places for interaction between people. An individual can choose three main ways to reduce the dissonance created by the difference of its opinion with the one of the other members of its group [24]: changing its opinion to agree with others in the group, influencing others to change their opinions, or joining a different, attitudinally more congenial group.
The first two suggest the basic rules governing the individual interactions. The third one can be linked to the local topology of the network surrounding the individual. The present paper studies a simulation model reproducing the coupled effect of these three strategies in order to better understand the link between the individual choices and the organization of the society into groups.

Social networks coupled to various reaction processes, have been intensively studied in the last decades [28]: from epidemics [26], [27], to malware diffusion in electronic technology [19], collective behaviors [16], innovation diffusion and opinion dynamics [3], [11].

Moreover, many opinion dynamic models have been proposed to study the spreading of opinions: some of these models describe opinion as a discrete Boolean choice, like the Voter model [4], [17] or the Sznajd model [29]. The first model describing continuous opinion interaction is known as the Deffuant model [5]. This model is based on the concept of bounded confidence: two individuals having their opinion less far than a threshold are going to have closer opinion after the interaction. Some different implementations of this model taking into account a rejection process [20] or a different type of tolerance threshold connected to the opinion [12] have been proposed in the last years. In our model we incorporate the Deffuant model to describe the opinion dynamics contact process.

The interest toward opinion dynamics increases coupling these phenomena with the investigation on the topological structure of social networks: different kinds of net- 
work topologies have been tested both to prove the robustness of the opinion dynamics models and for identifying preferential channels of opinion spreading [1].

Recently many works have been done regarding evolving network topology and their adaptation to the social background [14]: as people can influence each other to induce a change of mind, the difference of opinion on some very important topics can also lead to the breaking of a social contact. In other terms, since people prefer to be surrounded by persons sharing similar opinion, it is quite likely that the change of opinions due to the opinion dynamics' processes can lead to the change of the network structure. Two interesting analysis of the co-evolution of opinions and networks are [21] and [18]. In both these cases the opinion dynamics process takes place on a random graph and the connections among the agents are rewired in order to directly connect to someone with a similar opinion. In the two cases the formation of completely separate groups, sharing the same opinion, is observed at the end of the simulation. By the way the groups observed in these cases are an effect of the dynamics and are not a basic ingredient in its own construction. There is no impact of the group by itself on the dynamics of the individuals as we propose in the present work.

In this paper we start from the assumption that social groups exist a priori in the society: sociologists and network scientists agree on the fact that social networks usually present community based structures. Namely, analyzing networks at different scales, it is possible to identify groups of persons who are more interconnected than with the rest of society [13]. Many different algorithms have been designed to identify communities on large networks [10] and many models have been proposed to explain the mechanism leading to the formation of such underlying structures [25].

In this paper we study the reciprocal influence between opinion dynamics and group evolution. In the proposed model an individual can choose at each step of the simulation between changing its opinion interacting with one of its neighbours and changing its membership.

The interaction of the two different scales (individual and groups) has a twofold result. On one side the critical properties of the opinion dynamics contact process are changed since the group dynamics does not allow the formation of minor opinion clusters. We also notice that the group, considered as a subpopulation, is generally more consensual than the population itself. On the other side the superposition of the two dynamical process can generate interesting prototypical behaviours for the group structures in different points of the parameter space.

The model setup. - We build a network that incorporates the group structure. We consider $N$ agents and a fixed number $G$ of groups. Each person at the beginning chooses randomly the membership in one of the $G$ groups. The network is initially constructed linking together all the agents members of the same group. Moreover each person has also the possibility of being connected with someone that is not a member of its own group. Therefore, some other links between each agent and the agents outside its group are added with a probability $p_{\text {ext }}$. Since the group membership is randomly chosen, at the beginning all the groups have on average the same size $(S=N / G)$.

Together with the network structure we are interested in studying some kind of dynamical process on the network. We focus on an opinion dynamics process and therefore we need to initialize the opinion of the agents. We use a continuous opinion framework and we attribute to each agent a random opinion in the range $\vartheta_{i} \in[0,1]$. Since the opinion is initialized randomly, it results that the average opinion of each group, at the beginning, is around $O_{I} \sim$ 0.5 .

The aim of this paper is to study simultaneously the evolution of the network topology - and therefore the properties of the group structures - and the opinion dynamics process.

The opinion of a person, indeed, has an influence for its preferences about the connections: people prefer to be linked with someone with a similar opinion (homophily). On the other side, the opinion dynamics is a contact process that take place on the links of the social network and therefore it is influenced by the topology of the network. This double feedback is realized including in the model two dynamical modules: the first one concerns opinion dynamics on the network, and the other one the local changes in the network topology. At each time step each agent can choose with an equal probability which module to perform.

The dynamics of opinions. To model the opinion dynamics we used an extremely known model for opinion dynamics: the Bounded confidence (BC) model by Deffuant et al [5]. An agent, selected to perform opinion dynamics, decides to interact with one randomly chosen neighbour, whatever it is an external or an internal group link. According to the $\mathrm{BC}$, the two interacting agents $i$ and $j$ influence each other if their opinions differ from less than a fixed threshold $\varepsilon$. When they influence each other, their opinions become more similar:

if $\left|\vartheta_{i}(t)-\vartheta_{j}(t)\right|<\varepsilon\left\{\begin{array}{l}\vartheta_{i}(t+1)=\vartheta_{i}(t)+\mu\left(\vartheta_{j}(t)-\vartheta_{i}(t)\right) \\ \vartheta_{j}(t+1)=\vartheta_{j}(t)+\mu\left(\vartheta_{i}(t)-\vartheta_{j}(t)\right)\end{array}\right.$

where $\vartheta_{i}$ is the opinion of agent $i, \vartheta_{j}$ the opinion of agent $j$ and $\mu$ the speed coefficient. This model has been extensively studied and all the details of the dynamics are known. The BC model presents different behaviours according to different values of $\varepsilon$; in particular four types of bifurcations separating different types of behaviours have been identified in [2] and [23]: appearance of two minor clusters symmetrically from the central one at $\varepsilon \sim 0.5$ (transition from consensus to pluralism); creation of two major side clusters from the central one at $\varepsilon \sim 0.266$; separation of a minor central cluster at $\varepsilon \sim 0.222$; growth of the central cluster and shift to extremist positions of the 
two side clusters $\varepsilon \sim 0.182$.

In particular it has been showed in [9] that the transition between total consensus and pluralism at $\varepsilon=0.5$ is very robust according to the network topology: it remains the same if the dynamics happens on complete graphs, lattices, random graphs and scale free networks.

The dynamics of membership. The changes of the local topology of the networks are driven by the agents' decisions regarding the group membership. Following the dissonance theory of Festinger [8], we assume that an agent with an opinion very different from the average of its own group $\left(\left|\vartheta_{i}-O_{I}\right|>\varepsilon\right)$ can be uncomfortable and can decide to change group. The choice of the new group will be done between the set of groups on which he can retrieve some information, namely the groups in which he has some external connections. The choice of the new group $(J)$ happens with a probability:

$$
P_{i \rightarrow J}=\frac{1-\left|\vartheta_{i}(t)-O_{J}(t)\right|}{\sum_{J \supset j \in \mathcal{V}(i)}\left(1-\left|\vartheta_{i}(t)-O_{J}(t)\right|\right)}
$$

where $\mathcal{V}(i)$ is the neighbourhood of the agent $i$.

After the membership has changed, all the connections of the agent $i$ are re-initialized: it is connected to all the members of the new group and, with probability $p_{\text {ext }}$ with the agents outside the new group.

Results. - The model presents interesting results, both at the global level, where we can observe substantial differences with the Deffuant BC model, and regarding the evolution of the group structures. We will present the results in the following three sections.

Opinion Dynamics at population level. $\quad$ Figure 1 represents the density of opinion at the final state as a function of the parameter $\varepsilon$. This density has been calculated on 100 replications for each set of parameter values.

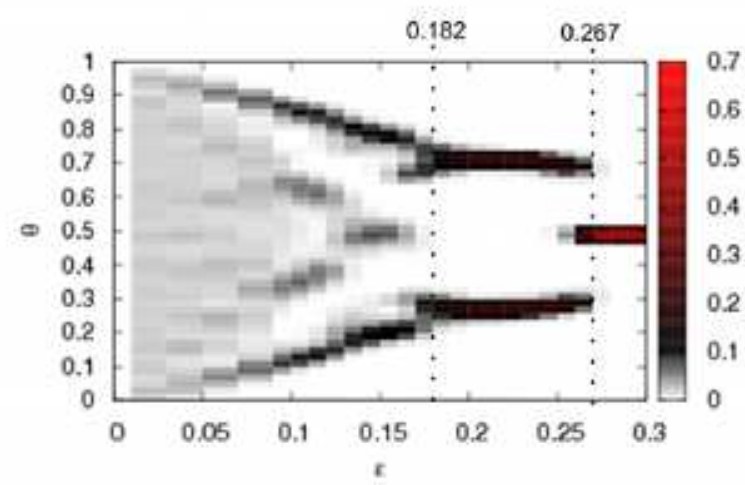

Fig. 1: Opinion density for different values of $\varepsilon$. The results concern a population of $N=5000$ individuals. The data are averaged on 100 realizations for each value of $\varepsilon$.

We can observe that, when the opinion dynamics is coupled with a co-evolutive network structure, the system presents a sudden transition between a state of consensus and a state of polarization of opinion (namely two major opinion clusters) at $\varepsilon_{c} \sim 0.27$.

Therefore the critical behaviour of the $\mathrm{BC}$ model on adaptive networks presents some differences with the traditional one studied by Ben Naim and Lorenz respectively in [2] and [23].

To better characterize the phase transition we use different realizations of the system varying the size of the system. In all the cases the ratio $\gamma=N / G$ is keep constant and also $p_{\text {ext }}$ is fixed in order to always maintain on average $L_{\text {out }}=5$ external connections.

Figure 2 displays the fraction of realizations with more than one opinion clusters for different sizes of the system. As the size increases the shape of the function approaches more and more to a step function around the critical value $\varepsilon_{c} \sim 0.27$. The transition, on the other side, happens at $\varepsilon_{c}=0.5$ if we switch off the membership dynamics [6]. This transition is also observed, even if it is not explained, in the model proposed in [21]. We argue that the variation of the transition threshold in the case of co-evolving networks is due to the lack, in this case, of the minor clusters in the $\varepsilon$-range between 0.267 and 0.5 .

As explained in [2], [22] and [23], in the usual BC model, when $\varepsilon$ is comprised between 0.267 and 0.5 , some individuals remain on the border of the attitude space, forming minor clusters. They have been "forgotten" by the others due to the high speed of the dynamics. In fact, to go from one extreme to a central opinion requires intermediate opinion dynamics steps with someone who has an opinion no more dissimilar than the bound; when the dynamics is fast, the central opinion quickly converge to the center, leaving the extremists isolated.

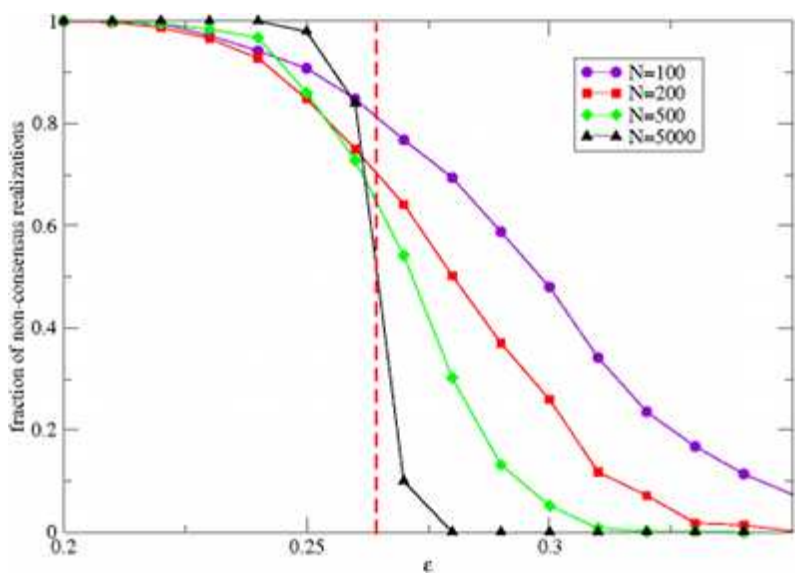

Fig. 2: Fraction of realizations with more than one opinion cluster for adaptive and static networks for different values of $N$. The data are averaged on 500 realizations.

In the case of groups, individuals can preferentially choose neighbours (preferring persons with similar opinion). This fact favours the interaction between more similar individual and creates a continuity of opinions, near the extremes, that allows the dynamics not to "forget" 
anybody. That is the reason why minor clusters do not exist in this "group" version of the Deffuant model.

To summarize this first section we can conclude that the structure of the opinion clusters observed on this adaptive topology is the same as in the classical BC model if we exclude the minor clusters. On the other side, the fact that minor clusters do not appear, shifts the consensus threshold to a lower value.

It can be interesting to approach this matter from the behaviour of the Hegselman and Krause model [15] which is also a bounded confidence model with a pseudo-group approach. Indeed, the group of an individual is dynamic in this instance and corresponds to all the individuals situated at an opinion distance around the individual of almost $\epsilon$. The individual interacting with its group members adopts their average opinion. This Hegselman and Krause version of the bounded confidence model also does not exhibit some minor clusters [23]. Indeed, the fact the individual interact with the whole group through all its members at the same time makes the convergence of the opinions more slow. It avoids, as it is the case when the dynamics of opinion occurs only in pairs of individuals (and not between an individual and the group), that some individuals remain on the extreme borders of the opinion space.

Opinon segregation at group level. In this section we analyze the opinion structure inside each groups. Figure 3 displays the average number of opinion clusters at population level compared to the group level.

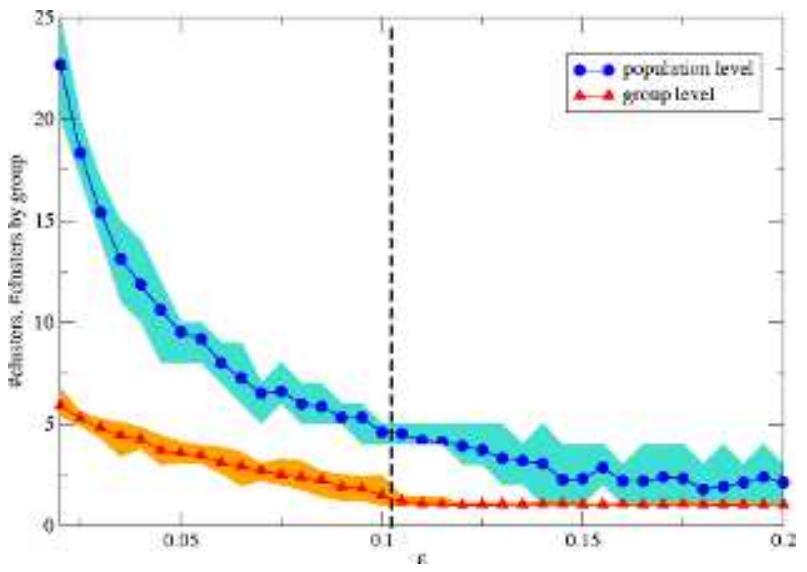

Fig. 3: Average number of opinion clusters at group level (red line) and at population level (blue line). The results concern a system of size $N=5000$. The data are averaged on 100 realizations of the system

We can observe that in general the number of cluster at population level is always higher than the number of clusters inside the groups. The opinion diversity is therefore always lower inside a group than inside the whole population. In particular, for $\varepsilon>0.1$, we can observe that all the groups contain at most one opinion cluster. It means that opinion result to be strongly segregated inside the groups: in each group the consensus is reached. From the point of view of the individuals, the fact that inside the group the opinion are uniform, means that all the individuals inside the groups are completely satisfied of their membership and membership dynamics is completely stable. An analysis of the range $\varepsilon<0.1$ is presented in [7].

Group hierarchy. At the moment of the initialization, all the groups have, on average, homogeneous sizes and the opinions are uniformly distributed inside the groups.

Figure 4 represents the group size distribution for different values of $\varepsilon$ at the end of the runs. For $\varepsilon=0.05$ the distribution is a stretched exponential with a long tail; this situation corresponds to a case where a giant macroscopic group (the tail of the distribution) is formed while all the other groups have a small size. For $\varepsilon=0.1$, the size distribution is described by a power law $\left(P(\sigma)=\sigma^{-\nu}\right)$ with exponent $\nu=0.18$, signifying that group sizes can exist at all the scales. Finally, for $\varepsilon=0.22$, the distribution observed is a Poissonian distribution with mean $\langle\sigma\rangle=N / G$.

This situation suggests the existence of a phase transition also regarding the group size around the critical value $\varepsilon_{c}(N=5000) \sim 0.1$ where the power law is observed.

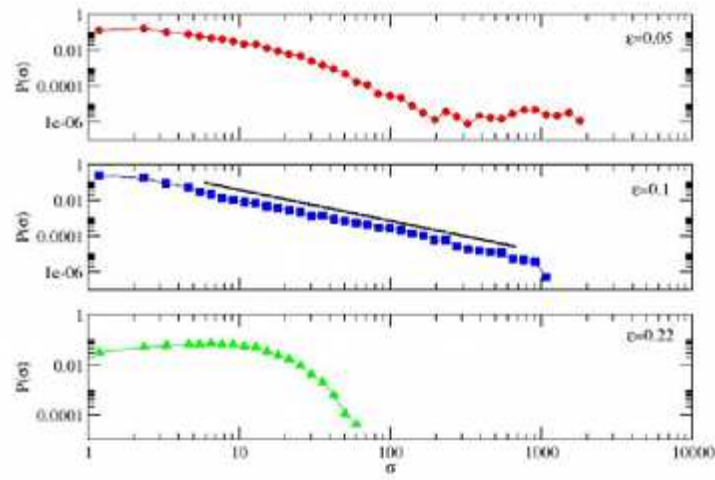

Fig. 4: Group size distribution for $\varepsilon=0.05,0.1,0.22$ and $N=5000$. The results are averaged on 500 realizations of the system.

Since in our model the number of groups and the number of agents are fixed since the beginning, the first moment of the size distribution $\langle\sigma\rangle=N / G$ is always conserved by the dynamics. However, once a group reaches size $\sigma=1$, it is automatically excluded from the dynamics. A good indicator to study the critical behaviour of the system is the average size, excluding the groups of size $\sigma=1,\langle\bar{\sigma}\rangle$. The measure $\langle\bar{\sigma}\rangle$ is maximal at the critical point. Since the exponent of the power law is $\nu<2$, the average size $\langle\bar{\sigma}\rangle$ diverges as $N \rightarrow \infty$. This fact allows us to perform some finite-size scaling analysis. The critical point $\varepsilon_{c}(N)$ is found in correspondence of the maximum of the function $\langle\bar{\sigma}\rangle(\varepsilon)$ for each size of the system. Looking to the limit of the function $\varepsilon_{c}(N)$ for $N \rightarrow \infty$, we can fix, in the thermodynamic limit $(N \rightarrow \infty), \varepsilon_{c} \sim 0.107$. 
Studying the scale relations for the larger group $\sigma_{\max }=$ $N^{-\alpha} \mathcal{F}\left(N\left(\varepsilon-\varepsilon_{c}\right)^{-\beta}\right)$ we can fix both $\alpha \sim \beta \sim 0.3$. This allows us to inscribe this process in the universality class of percolation on random graphs. A more fine analysis of the critical exponent should need a more exhaustive statistics and it is not the main interest of this paper where we want to provide a general description of the model.

The results obtained have some similarities to those presented in [18]. In this case the simulation procedure was quite different: first of all the observed quantity in the paper [18] are the separate components of the network obtained as the results of the simulation. In our case the groups are intrinsic dynamic objects defined a priori in the network structure such as the opinions. Moreover the groups are still connected among them at the end of the evolution and can contain an opinion richness higher than in the case of [18] as we will see in the following.

We will now provide a short heuristic explanation of the phenomena that lead to the creation of hierarchy in the group sizes. During a simulation, we distinguish two stages: a first one where people situated on the extrema tend to abandon their position in favour of the more extreme groups; a second one where the individuals with a more central opinion get together in one (or few) group. The first stage brings to an unbalance of the groups' average opinions: the concentration of extremists in some groups shifts the average opinion of such groups to one of the extremes. The second stage occurs when the opinion clusters are formed inside the group. At this time, since most of the groups have assumed an extremist average opinion in the previous phase, most of the individuals with a more central opinion remain unsatisfied by their own group. Thus, as they are now unable to change their opinion, they change groups using their external links, grouping in the few groups whose opinion remained neutral in the previous stage. A hidden preferential attachment mechanism is present in the group dynamics: if a group is a little larger in size than another, the individual has more external neighbours in this group. Thus the probability that this group is chosen increases with the size of the group.

Coming back to the considerations presented in the previous section, we can argue that the transition regarding the group sizes at $\varepsilon_{c} \sim 0.107$ is connected, at microscopic level, to the transition to consensus inside each group as observed in the figure 3 .

Conclusion. - We have proposed a model coupling the classical opinion dynamics of the bounded confidence model proposed by Deffuant with an adaptive network forming a community or group structure. At each step, an individual can decide if it changes groups or interact on its opinion with one of its internal or external neighbour. If it decides to look at the group level, it changes groups if its opinion is far from the average of its group from more than $\varepsilon$. If it is the case, it joins the group which has proportionally the closest average opinion from its own, and/or with which it is more linked. If it decides to interact with one of its neighbour, it becomes closer in opinion to it when its opinion and the one of the selectedto-interact neighbour are less distant from $\varepsilon$.

From the study of this coupled model, we discover some interesting behaviours compared to the known behaviour of the Deffuant bounded confidence model(BC). From the point of view of the critical behaviour, the coupled model exhibits a total consensus for an $\varepsilon$ value lower than the $\mathrm{BC}$ model. That is linked to the capacity of the coupled model to suppress the minor clusters positioned in the BC model on the extrema of the opinion space. In social psychology, groups are known as a source of cohesion and avoidance of the isolation. Thus, that is a very interesting fact that the introduction of groups in the $\mathrm{BC}$ model suppresses the isolated individuals.

From the point of view of the group structures two main observations can be pointed out: a hierarchy in the group structure is created and opinion tends to segregate inside different groups. Some groups, in fact, become larger while other decrease in size, sometimes until containing only one individual. This can be mainly explained by the fact that people unsatisfied by their group have a preferential external attachment to the larger groups and, possibly a preferential choice for the group to which they are more linked. Thus, when a group is a little bit larger due to the stochasticity of the model, it increases its probability to welcome unsatisfied people. More individuals come in, more larger it is and more probable the new arrivals in are. That sounds quite realistic. Indeed, a lot of people tend to change groups when they are in a dissonant situation and to join a larger group which appears more comfortable when it exists. Inside the groups, the internal consensus is reached for a large set of $\varepsilon$ values even if, looking at the population level, there are a lot of various opinion clusters. Then, each group does not only correspond to a subpopulation exhibiting the same behaviour than the whole population. In politics, we often see that a given opinion about an issue is the attribute of a given group. At the global level, each opinion present in the population correspond to a group's one. That tends to be not the truth when the opinion related issue is very important for people. In this case, the group level is less important for people and they prefer changing groups and remaining in the same opinion community.

Other aspects of this model are considered in [7] and a complete analysis about the influence of the number of groups, using a mathematical model, will be developed in a forthcoming paper.

\section{REFERENCES}

[1] F. Amblard and G. Deffuant. The role of network topology on extremism propagation with the relative agreement opinion dynamics. Physica A: Statistical Mechanics and its Applications, 343:725-738, 2004. 
[2] E. Ben-Naim, PL Krapivsky, and S. Redner. Bifurcations and patterns in compromise processes. Physica D: Nonlinear Phenomena, 183(3-4):190-204, 2003.

[3] C. Castellano, S. Fortunato, and V. Loreto. Statistical physics of social dynamics. Reviews of Modern Physics, 81(2):591-646, 2009.

[4] P. Clifford and A. Sudbury. A model for spatial conflict. Biometrika, 60(3):581, 1973.

[5] G. Deffuant, D. Neau, F. Amblard, and G. Weisbuch. Mixing beliefs among interacting agents. Advances in Complex Systems, 3(4):87-98, 2000.

[6] Gargiulo F. and S. Huet. Opinion dynamics on a group structured adaptive network. 2009.

[7] Gargiulo F. and S. Huet. How opinion dynamics generates group hierarchies. arXiv/1003.3560, 2010.

[8] L. Festinger. A Theory of Cognitive Dissonance. page 291, 1957.

[9] S. Fortunato. Universality of the Threshold for Complete Consensus for the Opinion Dynamics of Deffuant et al. International Journal of Modern Physics C, 15:1301-1307, 2004.

[10] S. Fortunato. Community detection in graphs. Physics Reports, 486:75-174, 2010.

[11] S. Galam. Sociophysics: A review of Galam models. International Journal of Modern Physics C, 19(3):409-440, 2008.

[12] F. Gargiulo and A. Mazzoni. Can extremism guarantee pluralism? JASSS, 11(4).

[13] M. Girvan and MEJ Newman. Community structure in social and biological networks. Proceedings of the National Academy of Sciences, 99(12):7821, 2002.

[14] T. Gross and B. Blasius. Adaptive coevolutionary networks: a review. Journal of the Royal Society Interface, $5(20): 259,2008$.

[15] R. Hegselmann and U. Krause. Opinion Dynamics and Bounded Confidence Models, Analysis and Simulation. Journal of Artificial Societies and Social Simulation, 5, 2002.

[16] D. Helbing, I. Farkas, and T. Vicsek. Simulating dynamical features of escape panic. Nature, 407(6803):487-490, 2000.

[17] R. Holley and TM Liggett. The Annals of Probability, 3.

[18] P. Holme and MEJ Newman. Nonequilibrium phase transition in the coevolution of networks and opinions. Physical Review E, 74(5):56108, 2006.

[19] H. Hu, S. Myers, V. Colizza, and A. Vespignani. WiFi networks and malware epidemiology. Proceedings of the National Academy of Sciences, 106(5):1318, 2009.

[20] S. Huet, G. Deffuant, and W. Jager. A Rejection Mechanism In 2d Bounded Confidence Provides More Conformity. Advances in Complex Systems, 11(4):529-549, 2008.

[21] B. Kozma and A. Barrat. Consensus formation on adaptive networks. Physical Review E, 77(1):16102, 2008.

[22] MF Laguna, G. Abramson, and D.H. Zanette. Minorities in a model for opinion formation. Complexity, 9(4):31-36, 2004.

[23] J. Lorenz. Continuous Opinion Dynamics Under Bounded Confidence:. a Survey. International Journal of Modern Physics C, 18:1819-1838, 2007.

[24] D. Matz and W. Woody. Cognitive dissonance in groups: The consequences of disagreement. Journal of personality and social psychology, 88(1):22-37, 2005.
[25] G. Palla, A.L. Barabási, and T. Vicsek. Quantifying social group evolution. NATURE-LONDON-, 446(7136):664, 2007.

[26] R. Pastor-Satorras and A. Vespignani. Epidemic spreading in scale-free networks. Physical review letters, 86(14):3200-3203, 2001

[27] R. Pastor-Satorras and A. Vespignani. Immunization of complex networks. Physical Review E, 65(3):36104, 2002.

[28] R. Pastor-Satorras and A. Vespignani. Evolution and structure of the Internet: A statistical physics approach. Cambridge Univ Pr, 2004.

[29] D. Stauffer. Sociophysics: the Sznajd model and its applications. Computer physics communications, 146(1):93-98, 2002 